\title{
Process Feasibility Investigation of Freezing Free Process
}

\author{
Tsuyoshi Nakamura ${ }^{\text {1) }}$, Ryoichi Takasu ${ }^{\text {1) }}$, Patrick Wong ${ }^{2)}$, Mireilli Maendhout ${ }^{2)}$, \\ 1) Tokyo Ohka Kogyo Co., Ltd. Tabata 1590, Samukawa, ,Kanagawa, Japan, \\ 2) IMEC Kapeldreef 75 B-3001 Leuven Belguim
}

\begin{abstract}
Double patterning technique with $193 \mathrm{~nm}$ immersion lithography is recognized as a potential candidate for $32 \mathrm{~nm} \mathrm{hp} \mathrm{node,} \mathrm{and} \mathrm{possibly} \mathrm{for} \mathrm{sub-32nm} \mathrm{hp} \mathrm{node}$ with recent. In this technique Litho-Process-Litho-Etch (LPLE) because of its simplicity is regarded as an attractive process. However, a workable application of this process has not yet been demonstrated.

Posi/Posi process that doesn't require freezing material has been investigated from the view-point of process simplification, and has been shown to be successful in printing images below $32 \mathrm{~nm}$ hp. Furthermore contact hole imaging by employing cross-line method has also been proven to be quite successful.

Here we present the results of our work on freezing free Posi/Posi process as applied to cross-lined contact hole, and results of the evaluation of high resolutions obtained from pitch splitting.
\end{abstract}

Key words: Double patterning, LPLE, Freezing free, 193nm immersion,

\section{Introduction}

Double patterning technique with $193 \mathrm{~nm}$ immersion lithography has been recognized as a promising candidate for $32 \mathrm{~nm}$ hp and beyond. However, the cost of double patterning can be considerably higher than that of single patterning lithography. Most chip manufacturers, therefore, are looking for simpler ways to do the job such as by employing double exposure or LPLE (Litho-Process-Litho-Etch).

We have been working to simplify double patterning by modifying the LPLE process that does not require a freezing. Elimination of freezing from the process could result in cost saving because additional materials and chambers would no longer be needed.

Our quest for LPLE without freezing led to the development of a Posi/Posi process which is the subject of this paper. The Posi/Posi process is the simplest among the double pattern techniques, ex- cept for the process of double exposure. And moreover, the Posi/Posi process is likely to cost less because of its simple structure of posi-resist film on posi-resist stack.

In recent days the chip manufacturers have also begun to focus on cross-line contact hole in addition to their currently employed pitch splitting dual line processing. Hence, we have investigated the feasibility of both pitch splitting and cross-line contact hole.

In this paper we present our test results to explore the feasibility of Posi/Posi process for pitch splitting and cross-line contact hole applications.

\section{Experiment}

2.1 Freezing free Posi/Posi Process flow

Freezing free Posi/Posi process involves application of posi-resist on posi-resist. A Posi/Posi process entails two areas of concerns. One is the mixing of 
$1^{\text {st }}$ and $2^{\text {nd }}$ resists and the other is exposing of $2^{\text {nd }}$ pattern on top of $1^{\text {st }}$ pattern. As reported before, the first concern can be resolved by application of an alcohol solvent for $2^{\text {nd }}$ resist. Because the solubility of the $1^{\text {st }}$ resist in alcohol solvent is quite low. The other concern can be addressed by devising methods to widen the gap between the activation energies and of the $1^{\text {st }}$ and the $2^{\text {nd }}$ resist functional groups. In case of the $2^{\text {nd }}$ exposed on the $1^{\text {st }}$ pattern, lower PEB for the $2^{\text {nd }}$ imaging can keep $1^{\text {st }}$ pattern, because activation energy of $1^{\text {st }}$ pattern is too high $[4,5]$. In this study, $150^{\circ} \mathrm{C}$ postbake after the $1^{\text {st }}$ imaging was found to be necessary in order to reduce the impact from the $2^{\text {nd }}$ imaging. As it appears from the FT-IR chart in Fig. 1, that no significant changes in cross-linking, and in polarity occur as a result of $150^{\circ} \mathrm{C}$ postbake. The difference between before and after $150^{\circ} \mathrm{C}$ postbake seems a peak at $1240 \mathrm{~cm}^{-1}$. According to GC-MS results presented in Fig. 2, the

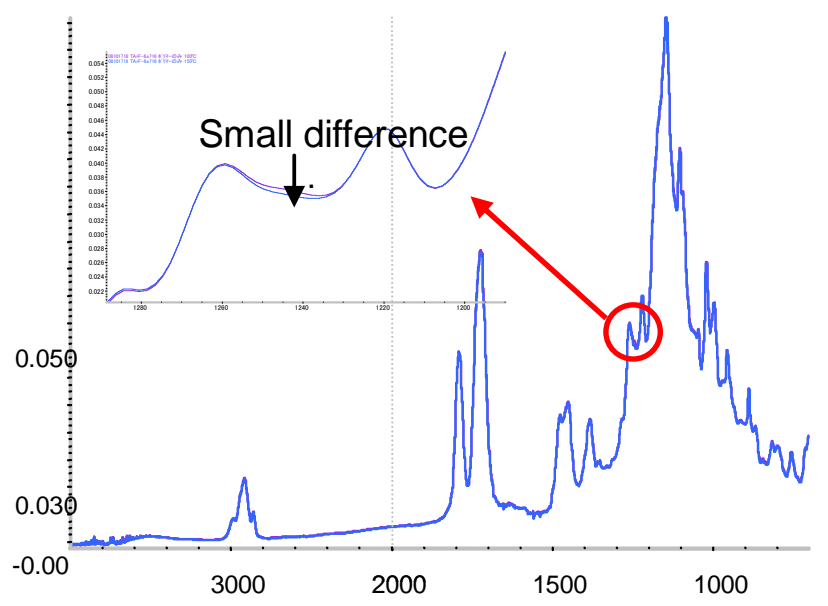

Fig.1 FT-IR chart before and after 150C postbake

$1^{\text {st }}$ resist film before $150^{\circ} \mathrm{C}$ postbake shows $1.6 \mathrm{wt} \%$ of PGMEA remaining in film. And after $150^{\circ} \mathrm{C}$ postbake no trace of PGEMA is seen. This means that postbake simply removes the solvent from the $1^{\text {st }}$ pattern. So, freezing free Posi/Posi process would require a high activation energy for the $1^{\text {st }}$ resist, and a low activation energy for the $2^{\text {nd }}$ resist by using an alcohol solvent, and by employing a $150^{\circ} \mathrm{C}$ postbake after the $1^{\text {st }}$ imaging. Actual process flow of Posi/Posi is shown in Fig. 3. In this work, we prepared TARF-Pi6-001 as the $1^{\text {st }}$ resist and TArF-PP006 as the $2^{\text {nd }}$ resist. TARF-Pi6-001 requires $120^{\circ} \mathrm{C}$ for $\mathrm{PAB}$, and $110^{\circ} \mathrm{C}$ for $\mathrm{PEB}$, both for 60s. TArF-PP006 requires $120^{\circ} \mathrm{C}$ for $\mathrm{PAB}$, and $90^{\circ} \mathrm{C}$ for PEB, both for 60 s also.

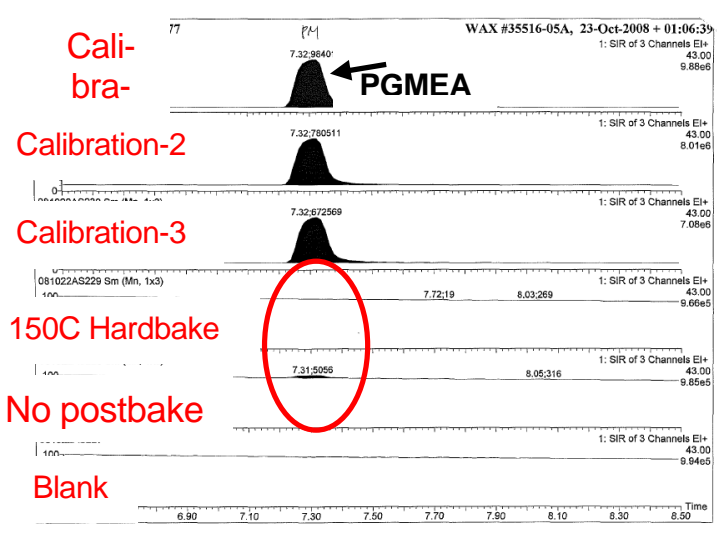

Fig.2 GC-MS of $1^{\text {st }}$ resist film w/ and w/o $150^{\circ} \mathrm{C}$

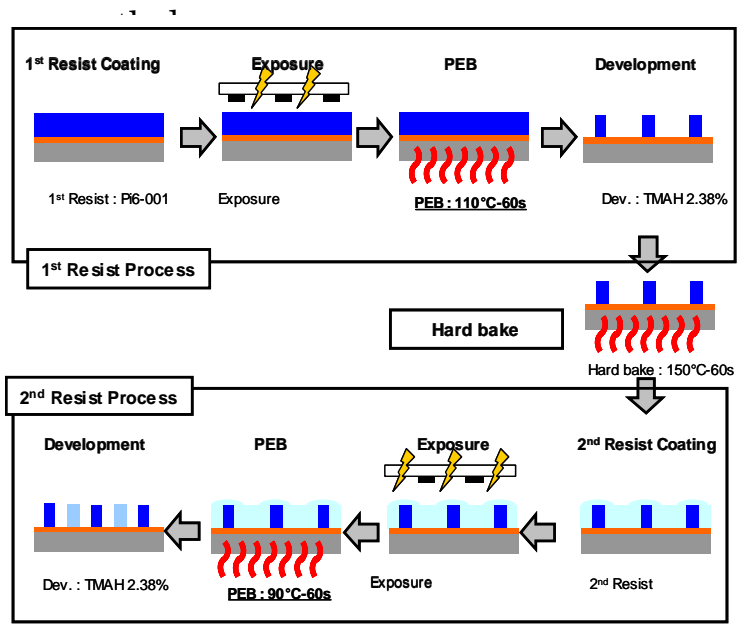

Fig. 3 Freezing free Posi/Posi process flow

\subsection{Pitch splitting dual line}

For imaging below 32nm hp dual line, Pi6-001 was coated on ARC29SR with $95 \mathrm{~nm}$ of thickness and PP006 was coated with 70nm of thickness. The Pi6-001 film was also covered with a top-coat. All wafers for pitch splitting tests were marked for the purpose of alignment. Both the $1^{\text {st }}$ and $2^{\text {nd }}$ resists were exposed under the same conditions. The exposure was made by ASML XT1900i with att-PSM. The Illuminations were set to 1.35 NA annular, and 1.0 NA dipole with polarization. Development was carried out with TMAH 2.38wt\% aq, using ECO-nozzle.

\subsection{Cross-line contact hole}

For cross-line contact hole imaging, the film thicknesses of both resists were set to be same as in case of pitch splitting. The illumination was set to 1.35NA dipole with polarization. Exposure tool and development procedure were same as in case of pitch splitting. An additional step here was to apply a 90degree roatation to the wafer at the $2^{\text {nd }}$ exposure 
as shown in Fig. 4. In addition, TArF-6a-trial 100nm and TArF-PP006 90nm combination was tested with S609B using 1.07NA dipole to read a cross-section profile.

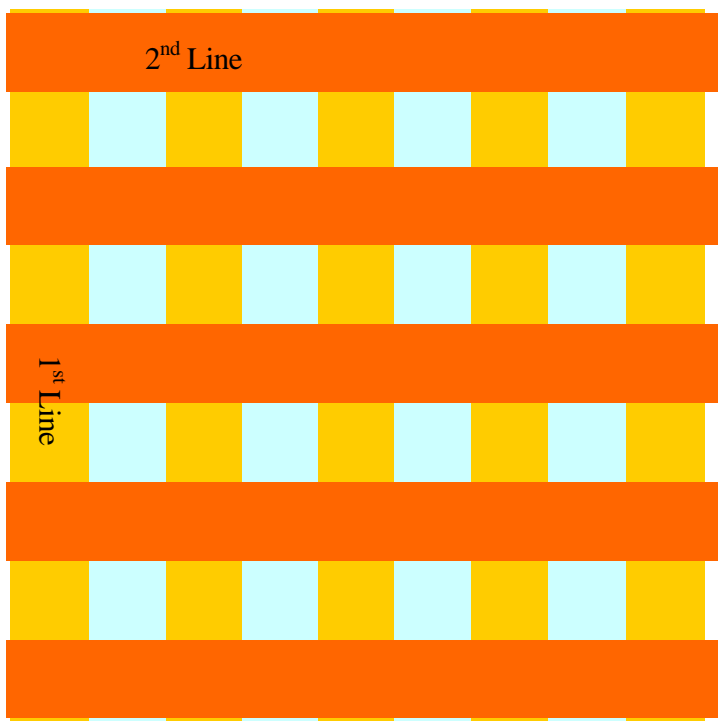

Fig. 4 Cross-line contact hole method

$1^{\text {st }}$ line $\quad 2^{\text {nd }}$ line

\section{Results and Discussion}

\subsection{Pitch splitting dual line}

At first, we proceeded to print 32nm hp dual line by using 1.35 NA annular. Mask sizes for both resists were $56 \mathrm{~nm}$ line with $128 \mathrm{~nm}$ pitch. From the imaging results, the $1^{\text {st }}$ resist $C D$ was $31.4 \mathrm{~nm}$ and the $2^{\text {nd }}$ resist $C D$ was $31.9 \mathrm{~nm}$. And after the imaging, the pattern height of the $1^{\text {st }}$ line was almost same as that of the $2^{\text {nd }}$ line (Fig. 5). This result pointed out that freezing free Posi/Posi process has good potential for controlling CD from litho down to the step of etching. We then studied the shift in the $1^{\text {st }}$ line $\mathrm{CD}$ as it went through the process. If the $2^{\text {nd }}$ resist process had an impact on the $1^{\text {st }}$ pattern, in case of the $1^{\text {st }}$ pattern CD change would have been larger. The results in Fig. 6, show that after the $1^{\text {st }}$ image, the $1^{\text {st }}$ line $C D$ was $34.5 \mathrm{~nm}$ and $C D$ change was almost zero after $150^{\circ} \mathrm{C}$ postbake. And then after the $2^{\text {nd }}$ imaging, the $1^{\text {st }}$ pattern $C D$ was $32.5 \mathrm{~nm}$. The $C D$ shift was thus less than $2 \mathrm{~nm}$. This negligible CD shift is indicative of a good CD control. Thus we studied the CD uniformity data for the $1^{\text {st }}$ and $2^{\text {nd }}$ lines on 32nm hp. The CD uniformity of the $1^{\text {st }}$ line was $1.45 \mathrm{~nm}(3 \sigma)$ and the uniformity of the $2^{\text {nd }}$ line was $2.74 \mathrm{~nm}$. These are considered to be quite good results for LPLE process. This means that Posi/Posi process is one of the promising candidates of LPLE

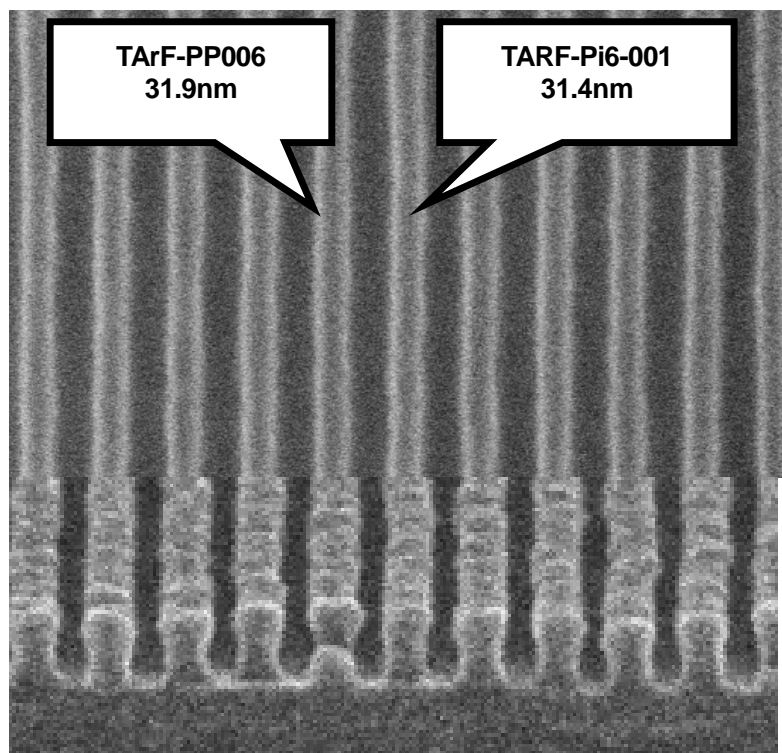

Fig. 5 32nm hp resolution result

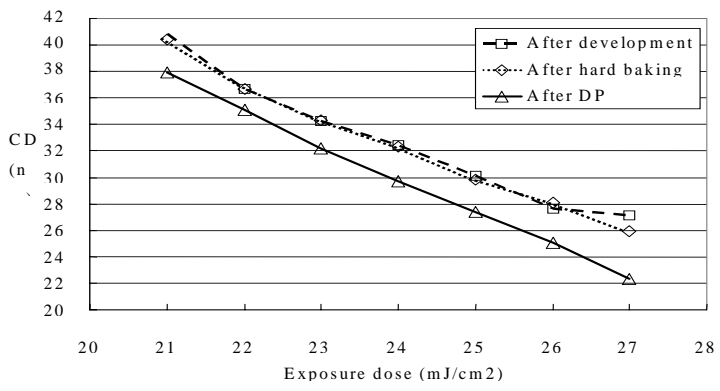

Fig. $6 \mathrm{CD}$ shift of $1^{\text {st }}$ pattern through process

in terms of CD control.

\subsection{Further resolution by pitch splitting}

To achieve further resolution down to 22nm hp, we evaluated same combination using NA 1.35 dipole. Figure 7 also shows that the $24 \mathrm{~nm}$ hp and $22 \mathrm{~nm}$ hp resolution images were obtained without any collapse. In this case $\mathrm{k} 1$ value was 0.15 .

\subsection{Cross-line contact hole}

\subsubsection{Aerial contrast calculation}

For contact hole application, cross-line contact

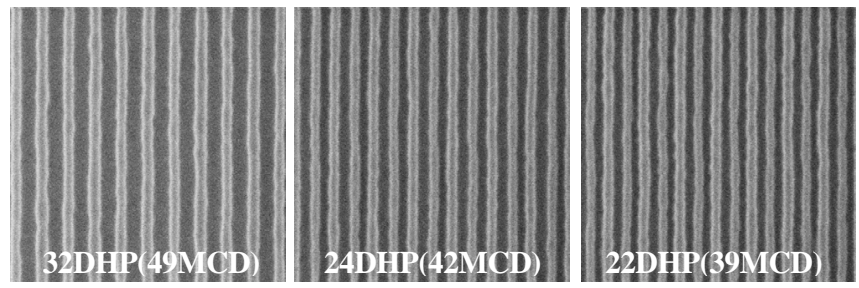

Fig. 7 Imaging result below 32nm hp

hole has become quite important for discussion and further investigations since the last year [6,7]. In 


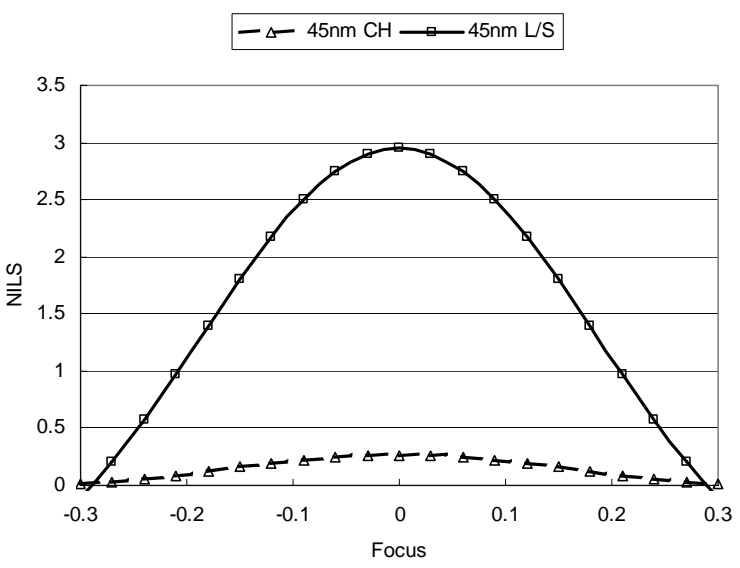

Fig. 8 NILS 45nm contact hole Annular, 45nm Line Dipole

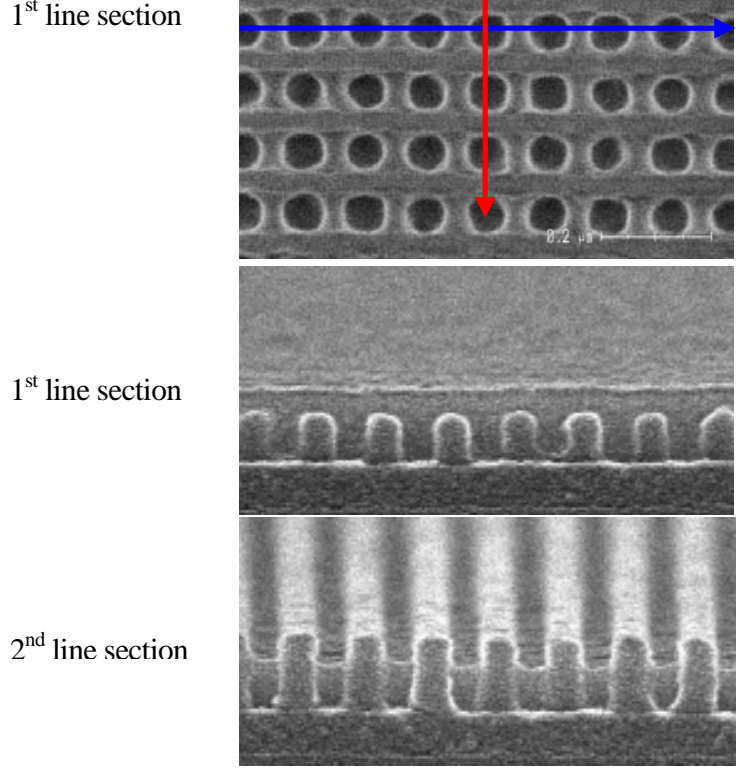

Fig. 9 55nm contact hole by cross-line

\section{5nm Mask/ 90nm Pitch}

6a716_14 mJ

PP006 $23 \mathrm{~mJ}$

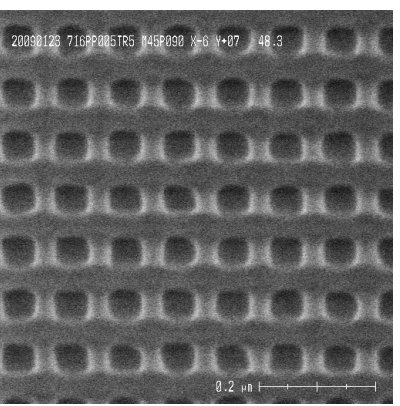

40nm Maskl 80nm Pitch

6a716_14 mJ PP006_29 mJ

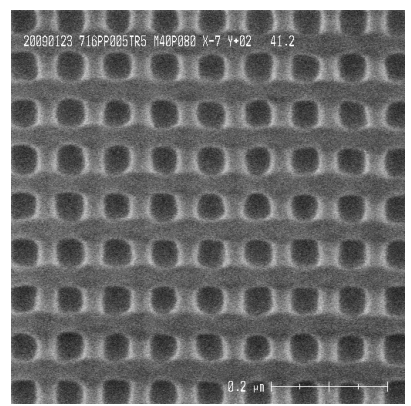

order to obtain $45 \mathrm{~nm}$ contact hole with $90 \mathrm{~nm}$ pitch, we calculated Normalized Illumination Log Slope (NILS) of cross-line method and of single imaging method. According to our calculation, cross-line method for contact hole has much higher NILS than single image method for contact hole has (Fig. 8). This means cross-line contact hole can have wider process latitude than conventional method can.

\subsubsection{Cross-line contact hole results}

To obtain smallest contact hole, a cross-line contact method requires line and space array with dipole illumination for the $1^{\text {st }}$ and $2^{\text {nd }}$ lines. The $2^{\text {nd }}$ line was exposed with 90degree rotation as mentioned above.

A 55nm cross-line contact hole image by using 1.07NA optics is shown in Fig. 9. We observed the cross-section profile for the $1^{\text {st }}$ and $2^{\text {nd }}$ patterns. The pattern heights in the $1^{\text {st }}$ and $2^{\text {nd }}$ resists were found to be different. This difference in heights is supposedly caused by the coatings on the topography from the $2^{\text {nd }}$ lithography. If the $2^{\text {nd }}$ resist film is kept thinner, the heights of the two patterns could be quite close [5]. For the smaller contact hole target, 6a716 and PP006 were exposed with 40nm line and space, and, then $40 \mathrm{~nm}$ contact hole $80 \mathrm{~nm}$ pitch pattern was obtained by using 1.35 NA dipole. Furthermore, $38 \mathrm{~nm}$ contact hole with $76 \mathrm{~nm}$ pitch was also obtained (Fig. 10). In this case, $\mathrm{k} 1$ value was down to 0.27 . In addition, DOF of $40 \mathrm{~nm}$ contact hole was studied and was found to be more than $0.24 \mu \mathrm{m}$. (Fig. 11)

For process feasibility, CD uniformity of cross-line contact hole was studied. A $40 \mathrm{~nm}$ contact hole array was imaged on a full wafer by using a combination of $6 a 716$ and PP006 with NA 1.35 dipole illumination. The result of CD uniformity indi-
Fig. 10 contact hole imaging with "Posi/Posi” process

\section{9nm Maskl 78nm Pitch}

PP006_32 mJ

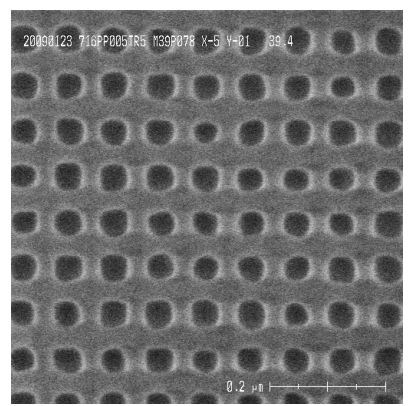

6 6716_16 mJ

\section{8nm Maskl 76nm Pitch}

$6 a 716 \_21 \mathrm{~mJ}$ PP006_38 mJ

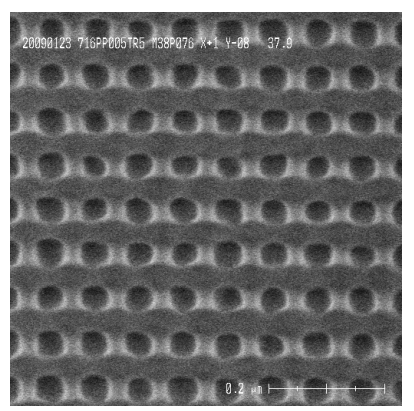




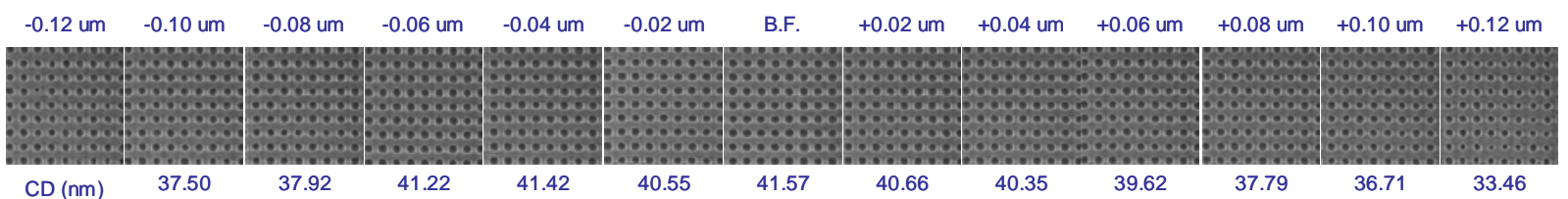

Fig. 11 DOF of 40nm CH with Posi/Posi process

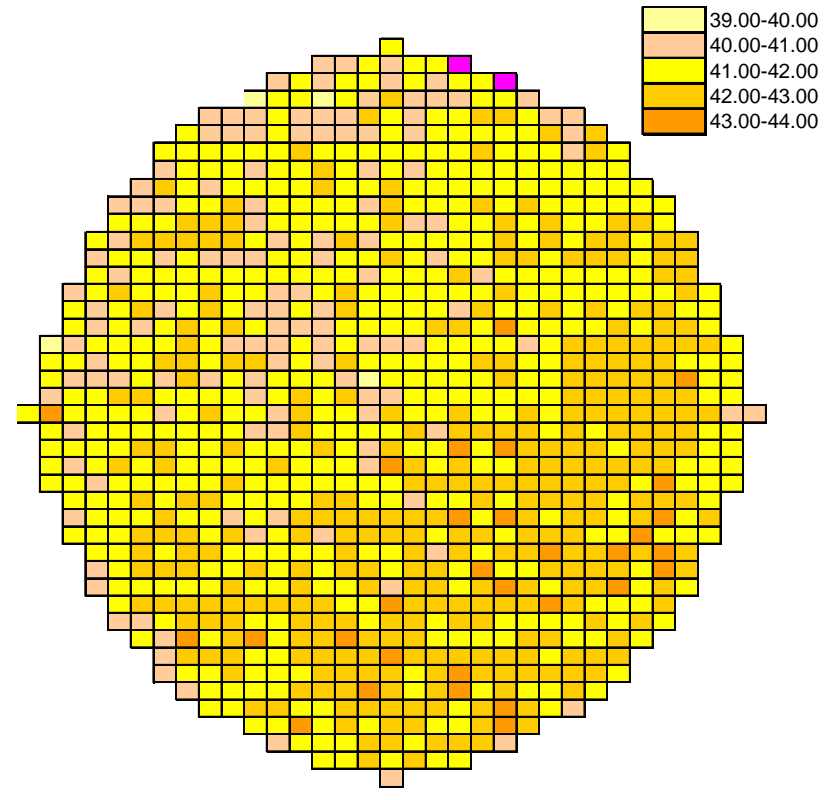

Fig. 12 CD uniformity of 40nm contact hole

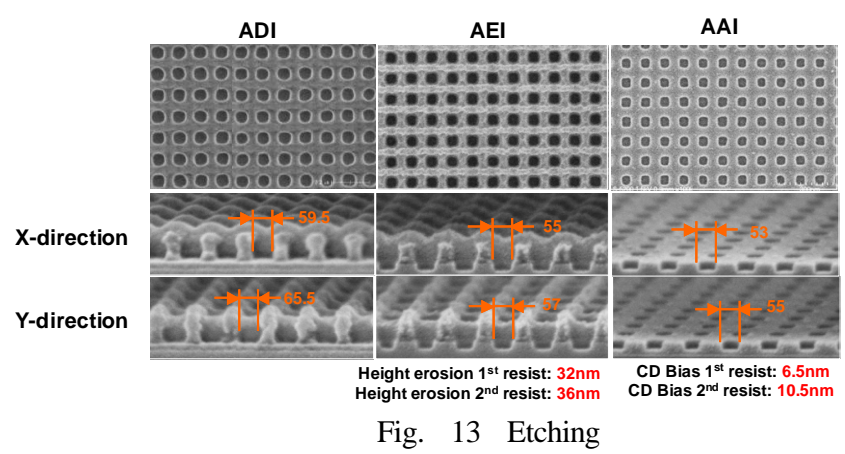

cated 2.01nm on intra-wafer (Fig. 12).

These results showed that cross-line contact hole method using freezing free Posi/Posi process is a strong candidate for shrinking the contact hole size, especially in contact hole array

\subsubsection{Etching results}

Etching capability of Posi/Posi cross-line contact hole was investigated. After development 60nm contact hole was imaged on SiON with ARC29SR by $1.07 \mathrm{NA}$ exposure. The etching was done by Hitachi U-8250 with CF4/CHF3 gas. By SiON etching, the $1^{\text {st }}$ resist height was eroded by $32 \mathrm{~nm}$ and the $2^{\text {nd }}$ resist height was eroded by $36 \mathrm{~nm}$ (Fig 13). CD biases through etching and ashing, were $6.5 \mathrm{~nm}$ and $10.5 \mathrm{~nm}$ for the $1^{\text {st }}$ and $2^{\text {nd }}$ lines. From these results, it is inferred that etching tolerance of the $1^{\text {st }}$ resist and $2^{\text {nd }}$ resist remain almost same

\section{Summary \& Future work}

A freezing free Posi/Posi process has been evaluated for its feasibility. The resolution capability of pitch doubling and cross-line contact hole were quite acceptable for 32nm node application and possible for sub-32nm node application. CD shift during the $2^{\text {nd }}$ imaging was small and CD uniformities of line and contact hole were also quite good. Moreover the etching tolerances of the $1^{\text {st }}$ and $2^{\text {nd }}$ resists were evaluated and found to be almost same. According to our results, freezing free Posi/Posi process has the potential for LPLE processing.

In future, the productivity of Posi/Posi process such as defectivity and lot-to-lot CD variation will be investigated.

\section{Acknowledgements}

The authors would like to thank IMEC engineers for helping on the evaluation of Posi/Posi process and TOK R\&D engineers for their useful discussions.

\section{References}

[1] Maenhoudt M. et al., "Double patterning scheme for sub- $0.25 \mathrm{k} 1$ single damascene structures at NA= 0.75, $\lambda=193 \mathrm{~nm}$ ", Proc. SPIE, 5754, (2005)

[2] Dusa M. et al., "Pitch doubling through dual patterning lithography challenges in integration and litho budgets”, Proc. SPIE, 6520, 65200G, 2007.

[3] Maenhoudt M. et al., "Alternative process schems for double patterning that eliminate the intermediate etch step” Proc. SPIE, 6924 (2008) 69240P

[4] Ando T. et al., "Pattern Freezing Free Litho-Litho-Etch Double Patterning” JJAP Microprocess and Nanotechnology 2008 proceeding.

[5] Ando T. et al., "Pattern Freezing Free Litho-Litho-Etch Double Patterning” Proc. SPIE, 7140 (2008)

[6] Hori. M et al., "Sub-40nm Half-Pitch Double Patterning with Resist Freezing Process" Proc. SPIE, 6923, (2007) 
[7] Tarutani S. et al., "Negative Tone Development Process for Double Patterning" $5^{\text {th }}$ International
Symposium of Immersion Lithography Extensions (2008) 